This item was submitted to Loughborough's Research Repository by the author.

Items in Figshare are protected by copyright, with all rights reserved, unless otherwise indicated.

\title{
Hepatic steatosis is associated with lower levels of physical activity measured via accelerometry
}

PLEASE CITE THE PUBLISHED VERSION

http://dx.doi.org/10.1002/oby.21058

\section{PUBLISHER}

(c) Wiley

\section{VERSION}

AM (Accepted Manuscript)

\section{PUBLISHER STATEMENT}

This work is made available according to the conditions of the Creative Commons Attribution-NonCommercialNoDerivatives 4.0 International (CC BY-NC-ND 4.0) licence. Full details of this licence are available at: https://creativecommons.org/licenses/by-nc-nd/4.0/

\section{LICENCE}

CC BY-NC-ND 4.0

\section{REPOSITORY RECORD}

Long, Michelle T., Alison Pedley, Joseph M. Massaro, Udo Hoffmann, Dale W. Esliger, Ramachandran S. Vasan, Caroline S. Fox, and Joanne M. Murabito. 2019. "Hepatic Steatosis Is Associated with Lower Levels of Physical Activity Measured via Accelerometry”. figshare. https://hdl.handle.net/2134/20851. 
Title: Hepatic Steatosis is Associated with Lower Levels of Physical Activity Measured via Accelerometry

Short title: Hepatic steatosis is associated with low physical activity

Authors: Michelle T. Long, $\mathrm{MD}^{1,2}$, Alison Pedley, $\mathrm{PhD}^{2}$, Joseph M. Massaro, $\mathrm{PhD}^{2,3}$, Udo Hoffmann, $\mathrm{MD}^{4}$, Dale W. Esliger, $\mathrm{PhD}^{5}$, Ramachandran S. Vasan, $\mathrm{MD}^{2,6}$, Caroline S. Fox, MD, $\mathrm{MPH}^{2,7}$ and Joanne M. Murabito, MD, $\mathrm{ScM}^{2,8}$.

${ }^{1}$ Division of Gastroenterology, Boston Medical Center, Boston University School of Medicine, Boston, MA

${ }^{2}$ National Heart, Lung, and Blood Institute's Framingham Heart Study, Framingham, MA

${ }^{3}$ Department of Mathematics and Statistics, Boston University, Boston, MA

${ }^{4}$ Radiology Department, Massachusetts General Hospital, Harvard Medical School, Boston, MA

${ }^{5}$ School of Sport, Exercise, and Health Sciences, Loughborough University, Loughborough, United Kingdom

${ }^{6}$ Section of Preventive Medicine, Department of Medicine, Boston University School of Medicine, Boston, MA

${ }^{7}$ Division of Endocrinology, Hypertension, and Metabolism, Brigham and Women's Hospital, Harvard Medical School, Boston, MA

${ }^{8}$ Section of General Internal Medicine, Department of Medicine, Boston University School of Medicine, Boston, MA

\section{Correspondence:}


Joanne M. Murabito, MD, ScM, Framingham Heart Study, National Heart, Lung, and Blood Institute, 73 Mount Wayte Avenue, Suite 2, Framingham, MA 01702, USA. Email: murabito@bu.edu

Tel.: 5089353461 Fax: 5086261262

\section{Specific Author Contributions:}

Study concept and design (MTL, RSV, CSF, JMM); acquisition of data (CSF,JMM, RSV); Analysis and interpretation of data (MTL, AP, JMMas, DWE, UH, RSV, CSF, $\mathrm{JMM}$ ); drafting of the manuscript (MTL); critical revision of the manuscript for important intellectual content (CSF, RSV, JMM); statistical analysis (AP, JMMas); administrative, technical, or material support (UH, DWE); study supervision (CSF,JMM). All authors approved of the final draft prior to submission.

\section{Funding support:}

This work was supported by the Boston University School of Medicine and the National Heart, Lung, and Blood Institute's Framingham Heart Study (contract N01-HC-25195), and the Division of Intramural Research of the National Heart, Lung, and Blood Institute. We received additional support from the NIH grant 1RO1-AG-047645. Dr. Long is supported in part by the Boston University Clinical and Translational Science Institute (grant UL1-TR000157).

\section{Conflicts of Interest:}

Alison Pedley is an employee of Merck. The other authors have no conflicts to report. 
What is already known about this subject?

- Non-alcoholic fatty liver disease (NAFLD) is a common condition with few available treatments.

- Guidelines recommend increasing physical activity in patients with NAFLD.

- Most prior studies utilize subjective measures of physical activity.

What does this study add?

- There is an inverse association between objectively measured physical activity, outside of a structured exercise intervention, and hepatic steatosis.

- Compliance with national physical activity guidelines may be associated with less hepatic steatosis. 


\section{Abstract:}

Objective: Prior studies on the association of physical activity (PA) and non-alcoholic fatty liver disease are limited by reliance on subjective measures of PA. We examined the association between objectively measured PA and hepatic steatosis defined by computed tomography (CT). Methods: We conducted a cross-sectional study of 1060 Framingham Heart Study participants who participated in the Multi-Detector CT 2 substudy and who underwent assessment of PA via accelerometry. Hepatic steatosis was estimated by liver attenuation, as measured by CT. We explored the relationship between liver attenuation and PA using multivariable regression models. Results: In multivariable-adjusted models, we observed an inverse association between PA and liver attenuation. Each 30 min/day increase in moderate-to-vigorous PA (MVPA) was associated with a reduced odds of hepatic steatosis $(O R=0.62, p<0.001)$. This association was attenuated and no longer statistically significant after adjustment for BMI $(\mathrm{OR}=0.77, \mathrm{p}=0.05)$ or VAT $(\mathrm{OR}=0.83, \mathrm{p}=0.18)$. Participants who met the national PA recommendations of engaging in $\geq 150$ minutes/week of MVPA, had the lowest odds of hepatic steatosis, even after adjusting for $\mathrm{BMI}(\mathrm{OR}=0.63, \mathrm{p}=0.007)$ or VAT (OR=0.67, $p=0.03$ ). Conclusions: There is an inverse association between PA and hepatic steatosis. Participants who met the national PA guidelines had the lowest prevalence of hepatic steatosis. 


\section{Introduction}

Non-alcoholic fatty liver disease (NAFLD) is the most common chronic liver disease in the United States.(1) NAFLD refers to a broad spectrum of liver injury from simple steatosis to nonalcoholic steatohepatitis (NASH) and cirrhosis. Large population based studies have demonstrated associations between NAFLD and increased allcause and liver-related mortality; $(2,3)$ however, there are no medical therapies available.

Currently, the cornerstone of treatment for NAFLD involves lifestyle interventions including increasing physical activity (PA).(4) The relationship between PA and NAFLD, independent of weight loss, has not been well characterized. In several small studies of exercise programs, liver fat content diminishes $(5,6,7)$ and intrahepatic triglyceride content decreases (8) with exercise independent of weight loss. A randomized controlled trial in obese patients found that the addition of physical activity to a dietinduced weight loss program promoted greater reductions in waist circumference and hepatic fat content.(9) The literature is still somewhat conflicting, as some studies show no improvement in histologic features of NASH with circuit exercise training.(10) The optimal dose of PA by intensity and duration for the prevention and treatment of NAFLD has not been well established. Prior population-based studies of the association of PA and NAFLD have been limited by the lack of objectively obtained PA data, with most studies relying on self-report, patient recall or leisure time only activities.(11, 12, 13, 14) The one population based study using accelerometer derived physical activity measurements found that participants with NAFLD were less physically active than participants without NAFLD. (15) However, this study defined NAFLD based on the 
Fatty Liver Index which does not incorporate imaging data and has been shown to have limited utility in detecting hepatic steatosis in obese patients.(16) In addition, there is emerging evidence that prolonged sedentary time, independent of PA, may be associated with cardiometabolic risk factors and overall mortality, but the associations with NAFLD has not been specifically evaluated. $(17,18,19)$

Thus, the purpose of the present study was to determine the association between objectively measured PA using accelerometry and hepatic steatosis in a large community-based sample. We hypothesized that participants with higher levels of PA would have a lower prevalence of hepatic steatosis. We also evaluated whether an association exists between sedentary time and hepatic steatosis. Finally, we assessed whether associations were stronger in participants who met the national PA guidelines of $\geq 150$ minutes of moderate-to-vigorous PA (MVPA) per week in total or accumulated in bouts of $\geq 10$ minutes.(20)

\section{Patients and Methods}

Additional methodological details are available in the online supplement.

\section{Study sample}

The Framingham Heart Study (FHS) is a multi-generational cohort study. Our sample was derived from a total of 3732 participants in the Third Generation Cohort and Omni 2 Cohort of the FHS who attended the second examination cycle (May 2008 to March 2011) when accelerometry was performed. Individuals were excluded from this analysis if they did not participate in the Multi-Detector CT 2 substudy $(n=2162)$ or their CT scans were not interpretable for liver attenuation or Visceral Adipose Tissue (VAT) 
$(n=14)$, they did not participate in accelerometry $(n=239)$ or had insufficient accelerometry data $(n=75)$, they had missing serum aminotransferase levels $(n=5)$, incomplete covariate data $(n=1)$ or significant alcohol use defined as $>7$ drinks per week for women and $>14$ drinks per week for men $(n=176)$, yielding a total sample of 1,060 participants. The institutional review boards of the Boston University Medical Center and Massachusetts General Hospital approved the study protocol.

Measurement of physical activity by accelerometer

During the second examination cycle between May 2008 to March 2011, Third Generation and Omni Cohort 2 participants were instructed to wear an omnidirectional accelerometer (Actical Model No. 198-0200-00; Philips Respironics, Bend, OR) on a waist-worn belt 24 hours/day for 8 days (to allow for 7 full days of wear time) as previously described.(21)

Multi-detector CT scan protocol and measurement of VAT and liver attenuation

Multi-detector CT scans were conducted in a substudy of FHS participants from September 2008 to December 2011 as previously described.(22) We defined hepatic steatosis as a liver phantom ratio of $\leq 0.33$ based our prior work.(23)

Covariate and baseline measurements

Covariate and baseline measurements were measured at the second examination cycle (May 2008 to March 2011) for the Third Generation and Omni Cohort 2 participants when accelerometry was also routinely performed.

Statistical analysis 
To describe the characteristics of the analysis population, we used means with standard deviations for continuous traits and percentages for categorical traits. Sexspecific, age-adjusted Pearson correlation coefficients between PA categories (sedentary, light, MVPA and MVPA bouts) and metabolic parameters were calculated. Multivariable logistic regression models were used to assess the cross-sectional association with PA (per 30 minutes/day increase in PA or compliance with national PA guidelines) and hepatic steatosis as measured by multi-detector CT scan. An additional multivariable logistic regression model examining the association between sedentary time and hepatic steatosis was performed. For each dependent variable, three separate models were calculated. Model 1 adjusted for age, sex, cohort (Third Generation vs Omni), current smoking status, alcohol use (drinks/week), accelerometer "wear time" in days; for the analysis examining the association between sedentary time and hepatic steatosis, we additionally adjusted for light PA and MVPA minutes/day. The second model contained the same covariates from model 1 and additionally adjusted for BMI. Model 3 adjusted for the covariates in model 1 and additionally adjusted for VAT. We also tested for effect modification by sex in the primary model.

To explore the association of PA and aminotransferase levels, multivariableadjusted linear regression models examining the association between log-adjusted ALT and AST per 30 minutes/day increase in PA were performed. ALT and AST were natural log-transformed to normalize their skewed distribution.

Since hepatic steatosis is associated with obesity, we further characterized the association between MVPA, hepatic steatosis and BMI, by stratifying participants by BMI category (normal BMI (BMI $\left.<25 \mathrm{~kg} / \mathrm{m}^{2}\right)$, overweight $\left(25 \mathrm{~kg} / \mathrm{m}^{2} \leq \mathrm{BMl}<30 \mathrm{~kg} / \mathrm{m}^{2}\right.$ ) 
and obese $\left(\mathrm{BMI} \geq 30 \mathrm{~kg} / \mathrm{m}^{2}\right)$ ). We used the t-test for differences in means to determine if there were differences in the mean MVPA among those with hepatic steatosis (LPR $\leq$ 0.33 ) and those without hepatic steatosis (LPR $>0.33$ ) across the BMI categories and for obese $\left(\mathrm{BMI} \geq 30 \mathrm{~kg} / \mathrm{m}^{2}\right)$ vs non-obese $\left(\mathrm{BMI}<30 \mathrm{~kg} / \mathrm{m}^{2}\right)$ participants.

A sensitivity analysis was conducted to assess the effect of using a less stringent definition of heavy alcohol use (defined as $>14$ drinks per week for women and $>21$ drinks per week for men) recommended by the American Association for the Study of Liver Diseases guidelines.(4)

Analyses were performed using SAS version 9.2 (SAS Institute, Cary, NC) software. A 2-tailed $\mathrm{P}$ value of $<0.05$ was considered significant.

\section{Results}

Study sample characteristics

The characteristics of the study sample (mean age $50.5 \pm 7.2 \mathrm{yrs}, 47.3 \%$ women), by sex, are depicted in Table 1. On average, participants were overweight (mean BMI $28.4 \pm 5.4 \mathrm{~kg} / \mathrm{m}^{2}$ ) and $22.0 \%$ of women and $32.0 \%$ of men had a LPR $\leq 0.33$ consistent with hepatic steatosis. On average, participants accumulated nearly 30 minutes/day of MVPA ( $26.6 \pm 20.4$ minutes of MVPA per day) and $14.5 \pm 14.3$ minutes of MVPA in bouts, defined as continuous MVPA of at least 10 minutes. Only $14.0 \%$ of women and $10.6 \%$ of men met the strict PA guideline of participating in $\geq 150$ minutes/week of MVPA in bouts, while about $50 \%$ of participants engaged in $\geq 150$ minutes/week of total MVPA. The average number of sedentary hours per day was $11.1 \pm 1.1$ (which excludes 8 hours of sleep) (Table 1). After adjusting for age, as 
expected, there was a moderate, positive correlation between more liver fat (- LPR) and VAT $(r=0.50, p<0.0001)$

Correlations between accelerometer derived physical activity and metabolic parameters MVPA minutes/day was associated with most adiposity traits in women and men (Table 2). Specifically, increasing MVPA minutes/day was associated with lower BMI in women only $(p<0.001)$, waist circumference, and VAT volume and a higher liver attenuation (less liver fat) and log AST (all p<0.05). Sedentary time was associated with VAT volume and log AST $(p<0.05)$ for women only.

Multivariable-adjusted regression models relating hepatic steatosis to physical activity

In multivariable-adjusted models, there was an inverse association between PA and hepatic steatosis (LPR $\leq 0.33)$ (Table 3$)$. Specifically, each 30 minute per day increase in MVPA was associated with a reduced odds of hepatic steatosis (OR= 0.62 , $\mathrm{p}<0.001)$. This association was attenuated and marginally significant after adjustment for $\mathrm{BMI}(\mathrm{OR}=0.77, \mathrm{p}=0.05)$ and no longer significant after adjustment for VAT $(\mathrm{OR}=$ $0.83, p=0.18$ ). Similar associations were found for MVPA performed in bouts. There was a significant sex interaction suggesting that associations between these factors was stronger in women compared to men (Table 3).

Multivariable-adjusted regression models relating hepatic steatosis to compliance with national physical activity guidelines 
For participants who met the national PA recommendations of engaging in $\geq 150$ minutes/week of MVPA, the odds of hepatic steatosis was lower compared to those not meeting these recommendations, even after adjusting for $\mathrm{BMI}(\mathrm{OR}=0.63, \mathrm{p}=0.007)$ or VAT (OR=0.67, $p=0.03)$ (Table 4). We found a significant sex interaction $(p=0.02)$. The magnitude of the association of reduced odds of hepatic steatosis among participants compliant with the national PA guidelines was greater in women than in men, and remained statistically significant after adjustment for BMI or VAT in women only.

Multivariable-adjusted regression models relating hepatic steatosis to sedentary time

There was not a statistically significant association between sedentary time and hepatic steatosis in any of the regression models (Supplementary Table 1).

Association between aminotransferase levels and physical activity

There were no associations with log ALT and increasing amounts of PA across all activity categories (Supplementary Table 2). However, for AST, for every 30 minute increase in MVPA minutes/day or MVPA bouts minutes/day and for those meeting the national PA guidelines, a significant positive association with log AST was observed.

Association of physical activity and hepatic steatosis by BMI category

The number of MVPA minutes/day accumulated per participant according to hepatic steatosis and BMI category are shown in Figure 1. Participants with hepatic 
steatosis performed the least amount of MVPA minutes/day within BMI categories; however, the difference was only statistically significant in the overweight BMI category $(p=0.02 ;$ MVPA normal weight hepatic steatosis vs non-hepatic steatosis $p=0.25 ;$ MVPA obese hepatic steatosis vs non-hepatic steatosis $p=0.48$ ). Non-obese participants (BMI $<30 \mathrm{~kg} / \mathrm{m}^{2}$ ) without hepatic steatosis $(\mathrm{n}=609)$ performed more MVPA minutes/day (29.3 $\mathrm{min} /$ day) compared to non-obese participants with hepatic steatosis $(\mathrm{n}=102)(22.6$ $\min /$ day $)(p=0.002)$.

Secondary Analysis

A secondary analysis adjusting the exclusion criteria to use a less stringent definition of heavy alcohol use(4) was performed and the results of multivariable regression models were essentially unchanged (data not shown).

\section{Discussion}

Principle findings

In this cross-sectional study in a large, unselected, community-based cohort, our findings are 3-fold. First, we observed that participants who engaged in higher levels of PA were less likely to have prevalent hepatic steatosis. Second, compliance with national PA guidelines was associated with the lowest odds of hepatic steatosis even in models adjusting for BMI. The magnitude of this association was stronger in women compared to men and remained statistically significant after adjusting for BMI or VAT in 
women only. Finally, there were no associations between sedentary time and hepatic steatosis.

In the context of the current literature

There have been a number of small studies in select populations which have demonstrated improvements in hepatic steatosis in participants of structured exercise and weight loss programs. $(5,6,7,8,9)$ Little is currently known about the association of physical activity, outside of structured interventions, and NAFLD. Prior studies evaluating the association between PA and NAFLD have largely relied on self-reported PA assessments and our findings are in line with this prior work.(11, 12, 13, 14) Selfreported PA data can be hampered by recall and response bias as well as the inability to accurately quantify PA levels. $(24,25)$ To our knowledge, there has been only one other population-based study investigating objectively-measured PA and NAFLD that was conducted with the National Health and Nutrition Examination Survey (NHANES) data using a surrogate measure of NAFLD, the fatty liver index.(15) This study found an association between NAFLD and lower PA in age- and sex-adjusted models. Our investigation advances the current literature by demonstrating that the association between hepatic steatosis and lower PA remains statistically significant after adjustments in a multivariable regression model, including BMI or VAT, but only for participants that engaged in $\geq 150$ minutes per week of MVPA. This finding may indicate that there is a threshold of MVPA that must be exceeded before there are significant effects on liver fat. Additionally, our investigation further examined sedentary time and found no association with hepatic steatosis. 
The current national guidelines for PA for all Americans recommend at least 150 minutes of moderate PA a week or 75 minutes of vigorous activity a week or a combination of both accrued in bouts of at least 10 minutes to improve and maintain health (20). There has been very little prior work specifically evaluating these guidelines in NAFLD. In a cohort of biopsy proven NAFLD, subjects who met vigorous activity recommendations had a significantly decreased adjusted-odds of NASH compared to those who did not meet the vigorous activity recommendations.(12) This association was not seen for subjects who met the moderate-intensity PA recommendations. The authors conclude that intensity of PA, specifically vigorous activity, may be more important than duration or total volume of activity for the prevention or treatment of $\mathrm{NASH}$. However, this study was limited by the use of self-reported leisure time only PA which tends to over-report MVPA and under-report sedentary time compared with objectively measured, accelerometer derived, PA measurements. $(25,26)$ The amount of vigorous activity that participants in this study reported is likely an over-estimate since $26 \%$ of the sample met the vigorous PA guidelines, while in our investigation and others using accelerometer derived PA measurements, only $5.5 \%$ of participants met the vigorous PA guidelines.(26) By using an accelerometer derived assessment of PA, we have evaluated an objective measure of adherence to the national PA guidelines.

We observed no association between sedentary time and hepatic steatosis which, to our knowledge, has not been previously demonstrated. Prior populationbased studies have shown lower mortality from diabetes, cardiovascular disease and all-cause mortality with less sedentary time after adjusting for self-reported PA.(17, 18, 19) There is evidence that interrupting sedentary time with 2 minute bouts of light or 
moderate activity every 20 minutes may decrease postprandial glucose and insulin levels in overweight and obese adults.(27) We did not evaluate the effects of interrupted versus continuous sedentary time on hepatic steatosis, but this might be an interesting area of future investigation.

\section{Potential mechanisms}

Evidence from animal and human studies supports a benefit of exercise on hepatic fat content as well as skeletal muscle and adipose tissue deposits.(28) In humans, exercise reduces plasma free fatty acids and decreases intrahepatic triglyceride content independent of weight loss.(5, 8) Additionally, exercise likely also enhances insulin sensitivity in skeletal muscle thereby reducing insulin resistance which is highly correlated with NAFLD.(29) Exercise may also contribute to differences in fatty acid uptake and utilization in the liver. Regular exercise increases the capacity for fatty acid uptake and oxidation within skeletal muscle which may help decrease the fatty acid burden in other tissues, including hepatocytes.(30) Furthermore, exercise may directly augment hepatic fat by altering insulin-mediated intracellular utilization of fatty acids.(31)

We found significant sex interactions for hepatic steatosis and MVPA accrued in bouts and compliance with national PA guidelines where the effects were stronger in women compared to men. It is well-established that women and men have differences in fat distribution, with women having relatively lower VAT and higher SAT compared to men.(32, 33) Additionally, increasing VAT and SAT volumes are associated with more adverse cardiovascular risk factors in women compared to men.(32) Women have greater free fatty acid concentration and turn over compared to men which suggests sex 
differences in adipose tissue lipolysis.(34) Prior population based studies have demonstrated sex differences in NAFLD prevalence. In a study which defined NAFLD based on ultrasound, men had a higher prevalence of NAFLD compared to women in adjusted models.(35) Additionally, in the Dallas Heart Study which measured hepatic triglyceride content on magnetic resonance imaging, non-Hispanic white men had a 2fold increased risk of fatty liver compared to non-Hispanic white women.(36) This increased risk in men was not attributable to differences in body weight or insulin sensitivity and was not demonstrated in other ethnic groups.(36) It is not known if differential effects of physical activity contribute to sex differences in hepatic steatosis. Prior studies evaluating sex differences on the effects of physical activity or exercise on fat metabolism are very limited. $(30,37)$ In one small study, women were found to oxidize proportionally more lipid and less carbohydrate during exercise compared to men both before and after a 7 week endurance training program.(38) It is possible that sex difference in exercise induced lipolysis may contribute to the overall stronger associations with PA and reduced prevalence of hepatic steatosis among women compared to men in our study.(31) More research on potential sex differences of PA on hepatic steatosis are needed to further understand this interaction.

Implications

The intensity, amount and duration of PA required for the prevention and treatment of NAFLD is an important outstanding question since lifestyle modification remains the cornerstone of treatment.(4) Currently, there is no standardized recommendation for PA in NAFLD patients. Although cross sectional, our investigation gives support for suggesting that compliance with the national PA guidelines of at least 
150 minutes of moderate PA per week may be associated with less hepatic steatosis. Without objective measures, individuals may over-estimate their own physical activity, with larger differences between self-reported and accelerometer measured MVPA with higher levels of activity.(25) In practice, it is possible that an individual may report that they meet the national PA guidelines; however, when their activity is measured objectively, they may no longer meet guideline recommendations.

\section{Strengths and limitations}

The major strengths of our investigation include the use of a well-established and objective measure of PA. By taking advantage of a well-characterized, communitybased sample, with standardized demographic, laboratory and anthropometric measurements, we are able to add to the current literature by adjusting for several important confounders in exploring the association with PA and hepatic steatosis. Additionally, our community based sample has not been selected for hepatic steatosis.

There are a number of important limitations to our investigation that deserve mention. First, this is a cross-sectional, observational study and therefore we cannot evaluate temporality and cannot make inferences about causality. Although accelerometry is a well-established measure of PA, because it is worn on the hip, it is has a limited ability to measure activities that are performed while the hip is stationary, such as swimming or bicycle riding, which may have resulted in non-differential misclassification, which would bias towards the null. Additionally, the temporally fixed sleep time appropriation methods may not fully represent the specific accelerometer wear patterns of the participants. An important assumption to acknowledge is that the majority of participants would be sleeping between the hours of 2200 and 0600 and we 
did not want this sedentary time to be counted with wakeful sedentary time. We estimated liver attenuation via CT imaging, which likely underrepresents the burden of hepatic steatosis in the population since CT imaging is non-specific and insensitive to mild steatosis compared to the gold standard of liver biopsy.(39) This may have led to non-differential misclassification, biasing our results towards the null. Therefore, our findings may underrepresent the true association between hepatic steatosis and physical activity. Additionally, since CT scan lacks the sensitivity to detect mild liver steatosis ( $<30 \%$ fat content), our study cannot evaluate the association between mild hepatic steatosis and physical activity. Since the LPR has a sensitivity of $70 \%$ for hepatic steatosis (23), this also has the potential to lead to non-differential misclassification, biasing our results towards the null. Additionally, CT imaging cannot accurately detect steatohepatitis so we are unable to determine the association of PA on fatty liver disease severity. Hepatic fat content can fluctuate rapidly with exercise and changes in weight. In this cross-sectional study, we are not able to measure the effects of PA and liver attenuation directly. We also lack information about viral hepatitis status and other chronic liver conditions which can cause the appearance of liver fat on CT scan. The seropositivity of hepatitis C is low in the general population (1-2\%) so the possibility of confounding because of chronic hepatitis $C$ in our population is low.(40) Additionally, our sample is overwhelming Caucasian so the generalizability of our findings to individuals of non-European ancestry is not known.

\section{Conclusion}

In conclusion, we observed an inverse association between MVPA and hepatic steatosis. Participants who met the national PA guideline recommendations of 
engaging in at least $150 \mathrm{~min} /$ week of MVPA had a lower odds of hepatic steatosis, compared to those not reaching guideline recommendations. Future longitudinal studies using objectively measured PA assessments are required to further explore the role of PA for the prevention and treatment of hepatic steatosis. 
Figure and Table Legends:

Table 1:

MVPA indicates moderate-to-vigorous physical activity; BMI, body mass index; ALT, alanine aminotransferase; AST, aspartate aminotransferase. Data are presented as the mean (SD) for continuous traits and percentage having that characteristic for categorical data.

*A bout of MVPA is defined as at least 10 minutes of continuous MVPA allowing for a 2 minute interruption.

Table 2:

MVPA indicates moderate-to-vigorous physical activity; BMI body mass index; VAT, visceral adipose tissue; ALT, alanine aminotransferase; AST, aspartate aminotransferase.

${ }^{*}$ P-value $<0.05$

**p-value $<0.01$

${ }^{* \star *} p$-value $<0.001$

${ }^{\dagger} \mathrm{A}$ bout of MVPA is defined as at least 10 minutes of continuous MVPA allowing for a 2 minute interruption.

Table 3:

BMI indicates body mass index; VAT, visceral adipose tissue

*Multivariable (MV) Model includes age, sex (in overall model only), cohort (Third Generation or Omni), accelerometer wear time (valid days of use), current smoking status and drinks per week.

${ }^{\star *} \mathrm{~A}$ bout of MVPA is defined as at least 10 minutes of continuous MVPA allowing for a 2 minute interruption.

Table 4:

BMI indicates body mass index; VAT, visceral adipose tissue

*Multivariable (MV) Model includes age, sex (in overall model only), cohort (Third Generation or Omni), accelerometer wear time (valid days of use), current smoking status and drinks per week.

${ }^{* *}$ Met MVPA Guidelines is defined as participating in $\geq 150$ minutes of moderate-tovigorous physical activity per week.

*** Met MVPA Bout Guidelines is defined as participating in $\geq 150$ minutes of moderateto-vigorous physical activity per week accrued in bouts of at least 10 minutes. 
Figure 1: Bar chart depicting the mean moderate-to-vigorous physical activity (MVPA) in minutes per day + standard error (SE) by Body Mass Index (BMI) categories according to presence or absence of hepatic steatosis. The BMI categories are defined as normal BMI (BMI $\left.<25 \mathrm{~kg} / \mathrm{m}^{2}\right)$, Overweight $\left(25 \mathrm{~kg} / \mathrm{m}^{2} \leq \mathrm{BMI}<30 \mathrm{~kg} / \mathrm{m}^{2}\right)$ and Obese $\left(\mathrm{BMI} \geq 30 \mathrm{~kg} / \mathrm{m}^{2}\right)$. No hepatic steatosis represents a normal Liver Phantom Ratio (LPR) $($ LPR $>0.33)$ and hepatic steatosis represents an abnormal LPR (LPR $\leq 0.33)$. MVPA normal weight hepatic steatosis vs non-hepatic steatosis $p=0.25$; MVPA overweight hepatic steatosis vs non- hepatic steatosis ${ }^{*} p=0.02$; MVPA obese hepatic steatosis vs non-hepatic steatosis $\mathrm{p}=0.48$. 


\section{References:}

1. Younossi ZM, Stepanova M, Afendy M, Fang Y, Younossi $Y$, Mir H, et al. Changes in the prevalence of the most common causes of chronic liver diseases in the United States from 1988 to 2008. Clinical gastroenterology and hepatology : the official clinical practice journal of the American Gastroenterological Association 2011;9: 524-530 e521; quiz e560.

2. Adams LA, Lymp JF, St. Sauver J, Sanderson SO, Lindor KD, Feldstein A, et al. The Natural History of Nonalcoholic Fatty Liver Disease: A Population-Based Cohort Study. Gastroenterology 2005;129: 113-121.

3. Stepanova M, Rafiq N, Makhlouf H, Agrawal R, Kaur I, Younoszai Z, et al. Predictors of All-Cause Mortality and Liver-Related Mortality in Patients with Non-Alcoholic Fatty Liver Disease (NAFLD). Digestive diseases and sciences 2013.

4. Chalasani N, Younossi Z, Lavine JE, Diehl AM, Brunt EM, Cusi K, et al. The diagnosis and management of non-alcoholic fatty liver disease: practice Guideline by the American Association for the Study of Liver Diseases, American College of Gastroenterology, and the American Gastroenterological Association. Hepatology 2012;55: 2005-2023.

5. Johnson NA, Sachinwalla T, Walton DW, Smith K, Armstrong A, Thompson MW, et al. Aerobic exercise training reduces hepatic and visceral lipids in obese individuals without weight loss. Hepatology 2009;50: 1105-1112.

6. Keating SE, Hackett DA, George J, Johnson NA. Exercise and non-alcoholic fatty liver disease: a systematic review and meta-analysis. Journal of hepatology 2012;57: 157-166.

7. Shojaee-Moradie F, Baynes KC, Pentecost C, Bell JD, Thomas EL, Jackson NC, et al. Exercise training reduces fatty acid availability and improves the insulin sensitivity of glucose metabolism. Diabetologia 2007;50: 404-413.

8. Sullivan S, Kirk EP, Mittendorfer B, Patterson BW, Klein S. Randomized trial of exercise effect on intrahepatic triglyceride content and lipid kinetics in nonalcoholic fatty liver disease. Hepatology 2012;55: 1738-1745.

9. Goodpaster BH, Delany JP, Otto AD, Kuller L, Vockley J, South-Paul JE, et al. Effects of diet and physical activity interventions on weight loss and cardiometabolic risk factors in severely obese adults: a randomized trial. JAMA : the journal of the American Medical Association 2010;304: 1795-1802.

10. Hickman IJ BN, Croci I, Chachay VS, Clouston AD, et al. A Pilot Randomised Study of the Metabolic and Histological Effects of Exercise in Non-alcoholic Steatohepatitis. J Diabetes Metab 2013;4: 1-10. 
11. Krasnoff JB, Painter PL, Wallace JP, Bass NM, Merriman RB. Health-related fitness and physical activity in patients with nonalcoholic fatty liver disease. Hepatology 2008;47: 1158-1166.

12. Kistler KD, Brunt EM, Clark JM, Diehl AM, Sallis JF, Schwimmer JB, et al. Physical activity recommendations, exercise intensity, and histological severity of nonalcoholic fatty liver disease. The American journal of gastroenterology 2011;106: 460-468; quiz 469.

13. Bae JC, Suh S, Park SE, Rhee EJ, Park CY, Oh KW, et al. Regular exercise is associated with a reduction in the risk of NAFLD and decreased liver enzymes in individuals with NAFLD independent of obesity in Korean adults. PloS one 2012;7: e46819.

14. Zelber-Sagi S, Nitzan-Kaluski D, Goldsmith R, Webb M, Zvibel I, Goldiner I, et al. Role of leisuretime physical activity in nonalcoholic fatty liver disease: a population-based study. Hepatology 2008;48: 1791-1798.

15. Gerber L, Otgonsuren M, Mishra A, Escheik C, Birerdinc A, Stepanova M, et al. Non-alcoholic fatty liver disease (NAFLD) is associated with low level of physical activity: a population-based study. Alimentary pharmacology \& therapeutics 2012;36: 772-781.

16. Borman MA, Ladak F, Crotty P, Pollett A, Kirsch R, Pomier-Layrargues G, et al. The Fatty Liver Index has limited utility for the detection and quantification of hepatic steatosis in obese patients. Hepatology International 2012;7: 592-599.

17. Wilmot EG, Edwardson CL, Achana FA, Davies MJ, Gorely T, Gray LJ, et al. Sedentary time in adults and the association with diabetes, cardiovascular disease and death: systematic review and meta-analysis. Diabetologia 2012;55: 2895-2905.

18. van der Ploeg HP, Chey T, Korda RJ, Banks E, Bauman A. Sitting time and all-cause mortality risk in 222497 Australian adults. Archives of internal medicine 2012;172: 494-500.

19. Henson J, Yates T, Biddle SJ, Edwardson CL, Khunti K, Wilmot EG, et al. Associations of objectively measured sedentary behaviour and physical activity with markers of cardiometabolic health. Diabetologia 2013;56: 1012-1020.

20. 2008 Physical Activity Guidelines for Americans. In: US Department of Health and Human Services (HHS) OoDPaHP (ed). www.health.gov/paguidelines, 2008.

21. Glazer NL, Lyass A, Esliger DW, Blease SJ, Freedson PS, Massaro JM, et al. Sustained and shorter bouts of physical activity are related to cardiovascular health. Medicine and science in sports and exercise 2013;45: 109-115. 
22. Roseman DA, Hwang S-J, Manders ES, O'Donnell CJ, Upadhyay A, Hoffmann U, et al. Renal Artery Calcium, Cardiovascular Risk Factors, and Indexes of Renal Function. The American journal of cardiology 2014;113: 156-161.

23. Speliotes EK, Massaro JM, Hoffmann U, Vasan RS, Meigs JB, Sahani DV, et al. Fatty liver is associated with dyslipidemia and dysglycemia independent of visceral fat: the Framingham Heart Study. Hepatology 2010;51: 1979-1987.

24. Prince SA, Adamo KB, Hamel ME, Hardt J, Connor Gorber S, Tremblay M. A comparison of direct versus self-report measures for assessing physical activity in adults: a systematic review. The international journal of behavioral nutrition and physical activity 2008;5: 56.

25. Dyrstad SM, Hansen BH, Holme IM, Anderssen SA. Comparison of Self-reported versus Accelerometer-Measured Physical Activity. Medicine and science in sports and exercise 2014;46: 99-106.

26. Tucker JM, Welk GJ, Beyler NK. Physical activity in U.S.: adults compliance with the Physical Activity Guidelines for Americans. American journal of preventive medicine 2011;40: 454-461.

27. Dunstan DW, Kingwell BA, Larsen R, Healy GN, Cerin E, Hamilton MT, et al. Breaking up prolonged sitting reduces postprandial glucose and insulin responses. Diabetes care 2012;35: 976-983.

28. Bradley RL, Jeon JY, Liu FF, Maratos-Flier E. Voluntary exercise improves insulin sensitivity and adipose tissue inflammation in diet-induced obese mice. American journal of physiology Endocrinology and metabolism 2008;295: E586-594.

29. Mikus CR, Rector RS, Arce-Esquivel AA, Libla JL, Booth FW, Ibdah JA, et al. Daily physical activity enhances reactivity to insulin in skeletal muscle arterioles of hyperphagic Otsuka Long-Evans Tokushima Fatty rats. Journal of applied physiology 2010;109: 1203-1210.

30. Johnson NA, George J. Fitness versus fatness: moving beyond weight loss in nonalcoholic fatty liver disease. Hepatology 2010;52: 370-381.

31. Solomon TP, Haus JM, Marchetti CM, Stanley WC, Kirwan JP. Effects of exercise training and diet on lipid kinetics during free fatty acid-induced insulin resistance in older obese humans with impaired glucose tolerance. American journal of physiology Endocrinology and metabolism 2009;297: E552-559. 
32. Fox CS, Massaro JM, Hoffmann U, Pou KM, Maurovich-Horvat P, Liu CY, et al. Abdominal visceral and subcutaneous adipose tissue compartments: association with metabolic risk factors in the Framingham Heart Study. Circulation 2007;116: 39-48.

33. Pou KM, Massaro JM, Hoffmann U, Vasan RS, Maurovich-Horvat P, Larson MG, et al. Visceral and subcutaneous adipose tissue volumes are cross-sectionally related to markers of inflammation and oxidative stress: the Framingham Heart Study. Circulation 2007;116: 12341241.

34. Koutsari C, Basu R, Rizza RA, Nair KS, Khosla S, Jensen MD. Nonoxidative free fatty acid disposal is greater in young women than men. The Journal of clinical endocrinology and metabolism 2011;96: 541-547.

35. Lazo M, Hernaez R, Eberhardt MS, Bonekamp S, Kamel I, Guallar E, et al. Prevalence of nonalcoholic fatty liver disease in the United States: the Third National Health and Nutrition Examination Survey, 1988-1994. American journal of epidemiology 2013;178: 38-45.

36. Browning JD, Szczepaniak LS, Dobbins R, Nuremberg P, Horton JD, Cohen JC, et al. Prevalence of hepatic steatosis in an urban population in the United States: impact of ethnicity. Hepatology 2004;40: 1387-1395.

37. Blaak E. Gender differences in fat metabolism. Current opinion in clinical nutrition and metabolic care 2001;4: 499-502.

38. Carter SL, Rennie C, Tarnopolsky MA. Substrate utilization during endurance exercise in men and women after endurance training. American journal of physiology Endocrinology and metabolism 2001;280: E898-907.

39. Park SH, Kim PN, Kim KW, Lee SW, Yoon SE, Park SW, et al. Macrovesicular hepatic steatosis in living liver donors: use of CT for quantitative and qualitative assessment. Radiology 2006;239: 105-112.

40. Ioannou GN, Boyko EJ, Lee SP. The prevalence and predictors of elevated serum aminotransferase activity in the United States in 1999-2002. The American journal of gastroenterology 2006;101: 76-82. 
Supplementary Material

\section{Patients and Methods}

Study Sample

The Framingham Heart Study is a multi-generational cohort study. Beginning in 1971, the offspring and spouses of the offspring were enrolled in the Offspring Cohort. Between 2002 and 2005, the grandchildren of the original cohort and children of the Offspring cohort were enrolled in the Third Generation Cohort.(1) Starting in 1994, in order to reflect the growing ethnic diversity of the community, the Omni Cohort, which consists of minority residents of Framingham, MA and surrounding towns, was recruited. A second Omni Cohort was recruited between 2003 and 2005, with examinations paralleling that of the Third Generation Cohort. Between September 2008 and December 2011, Offspring, Third Generation and Omni 2 Cohort participants were enrolled in the Multi-Detector Computed Tomography 2 substudy and underwent chest and abdominal computed tomographic (CT) scanning. The median length of time between the CT scans and measurement of PA was 0.86 months with an interquartile range of 0.03 months to 4.21 months.

Measurement of physical activity by accelerometer

Participants were asked to remove the accelerometer only when bathing. At the end of the 8 day period, participants mailed the monitor to the FHS clinic. Data was visually screened for spurious data by FHS clinical staff. Standard quality control metrics were applied to the data and only data from participants with a minimum of 5 of 7 days of "valid" wear time were included in analyses as noted above. A "valid" wear day was defined as a minimum of 10 hours of wear time.(2) We used algorithms 
developed to process raw activity data from the Actical accelerometer to define sedentary, light, and MVPA minutes using analytical software as previously described (Kinesoft, version 3.3.63; KineSoft, Loughborough, UK).(3, 4) Briefly, time spent in each PA category is based on the accelerometer measured "counts" per minute which are calibrated to correspond to metabolic equivalents (METs) based on prior studies.(24) Light-intensity activity is defined as $>200$ counts/minute and $<3$ METs, moderate-intensity activity as between 3-6 METs and vigorous-intensity activity as $>6$ METs. $(24,25)$ Sedentary time is defined as $\leq 200$ counts/minute. The device was considered not worn if there were 60 minutes of consecutive zero counts allowing for 12 minute interruptions of non-zero counts.(25) Since the accelerometer was worn for 24 hours/day in order to maintain a high level of adherence with wearing the device, sleep time was defined empirically as between the hours of 2200 hours and 0600 hours and sedentary activity was not considered during this time period. All other count data contributed to wear time. Because the total time spent at vigorous-intensity activity was so small, moderate and vigorous-intensity minutes were summed to obtain the total MVPA time. Based on the national PA guidelines for Americans which recommends that all adults participate in at least 150 minutes of MVPA per week with activity accumulated in bouts of $\geq 10$ minutes (24), we additionally defined a bout of MVPA as at least 10 minutes of continuous MVPA, allowing for 2-minute interruptions to simulate conditions such as slowing a jog for a traffic light as has been done in prior research.(25) For this investigation, we defined compliance with the national PA guidelines as participating in $\geq 150$ minutes of MVPA over 5 to 7 days in bouts or in total MVPA, regardless of bout duration. 
Multi-detector CT scan protocol and measurement of VAT

For the CT scans, participants were placed in the supine position and twenty-five contiguous 5-mm-thick slices $(120 \mathrm{kVp}, 400 \mathrm{~mA}$, gantry rotation time $500 \mathrm{~ms}$, and table feed 3:1) covering $125 \mathrm{~mm}$ above S1 were obtained using an 8-slice multi-detector abdominal CT scanner (LightSpeed Ultra, General Electric, Milwaukee, WI). A calibration phantom (Image Analysis, Lexington, KY) with a water equivalent compound (CT-Water, Light Speed Ultra; General Electric, Milwaukee, WI) and calcium hydroxyapatite at 0,75 , and $150 \mathrm{mg} / \mathrm{cm}^{3}$ was placed under each participant and was visualized on each image obtained. Three areas from the liver, two areas from the spleen and one from the external phantom were measured and averaged to create liver spleen ratios and liver phantom ratios (LPR). The intra-class correlation coefficient was 0.99 on a subset of 50 randomly selected participants. Because the spleen was not visualized on all scans, the liver phantom ratio was used as the indexed standard.

VAT and subcutaneous adipose tissue (SAT) were measured using an image display window of -195 to -45 Hounsfield Units $(\mathrm{HU})$ and a window center of $-120 \mathrm{HU}$ to identify pixels containing fat. The VAT compartment was separated from the SAT compartment by a single reader who manually traced the muscular abdominal wall separating these depots. VAT and SAT volumes were subsequently quantified using a semiautomatic segmentation technique at a dedicated offline workstation (Aquarius 3D Workstation; TeraRecon, San Mateo, CA) as described.(5) The interclass correlation coefficients between two independent readers on a subset of 100 randomly selected participants were 0.992 and 0.997 for VAT and SAT, respectively. 
Covariate and baseline measurements

Serum alanine aminotransferase (ALT) and aspartate aminotransferase (AST) levels were obtained from fasting morning samples using an automated Roche method (Roche cobas 501). Data on ethnicity was obtained from self-administered questionnaires, while alcohol use and smoking status were assessed on the basis of physician-administrated questionnaires. Alcohol use was recorded as drinks per week or drinks per month. Participants were considered current smokers if they had smoked at least one cigarette per day in the year preceding the FHS examination.

Anthropometric measurements were obtained by trained technicians using standard protocols for measuring height, weight and waist circumference. BMI was defined as weight $(\mathrm{kg}) /$ height $^{2}\left(\mathrm{~m}^{2}\right)$.

References:

1. Splansky GL, Corey D, Yang Q, Atwood LD, Cupples LA, Benjamin EJ, et al. The Third Generation Cohort of the National Heart, Lung, and Blood Institute's Framingham Heart Study: design, recruitment, and initial examination. American journal of epidemiology 2007;165: 1328-1335.

2. Troiano RP, Berrigan D, Dodd KW, Masse LC, Tilert T, McDowell M. Physical activity in the United States measured by accelerometer. Medicine and science in sports and exercise 2008;40: 181-188.

3. Glazer NL, Lyass A, Esliger DW, Blease SJ, Freedson PS, Massaro JM, et al. Sustained and shorter bouts of physical activity are related to cardiovascular health. Medicine and science in sports and exercise 2013;45: 109-115. 
4. Heil DP. Predicting activity energy expenditure using the Actical activity monitor. Research Quarterly for Exercise and Sport 2006;77: 17.

5. Fox CS, Massaro JM, Hoffmann U, Pou KM, Maurovich-Horvat P, Liu CY, et al. Abdominal visceral and subcutaneous adipose tissue compartments: association with metabolic risk factors in the Framingham Heart Study. Circulation 2007;116: 39-48. 
Supplementary Table 1: Multivariable-adjusted logistic regressions examining the association between 30 minutes/day increment of sedentary time and hepatic steatosis (Liver Phantom Ratio $\leq 0.33$ ).

\begin{tabular}{|c|c|c|c|c|c|c|c|}
\hline & \multicolumn{2}{|l|}{ Women $(n=501)$} & \multicolumn{2}{|l|}{ Men $(n=559)$} & \multicolumn{2}{|l|}{ Overall $(n=1060)$} & \multirow{2}{*}{$\begin{array}{l}\text { P Value } \\
\text { for Sex } \\
\text { Interaction }\end{array}$} \\
\hline & OR (95\% Cl) & $\begin{array}{l}\mathrm{P} \\
\text { Value }\end{array}$ & OR (95\% Cl) & $\begin{array}{l}\mathrm{P} \\
\text { Value }\end{array}$ & OR $(95 \% \mathrm{Cl})$ & $\begin{array}{l}\mathrm{P} \\
\text { Value }\end{array}$ & \\
\hline $\begin{array}{l}\text { Sedentary } \\
\text { (min/day) }\end{array}$ & & & & & & & 0.70 \\
\hline $\begin{array}{l}\text { Multivariable } \\
\text { (MV) Model* }\end{array}$ & $0.98(0.88-1.08)$ & 0.64 & $1.0(0.92-1.09)$ & 0.99 & $0.99(0.93-1.06)$ & 0.76 & \\
\hline $\mathrm{MV}+\mathrm{BMI}$ & $1.0(0.88-1.13)$ & 0.98 & $1.03(0.93-1.13)$ & 0.62 & $1.02(0.94-1.10)$ & 0.67 & \\
\hline $\mathrm{MV}+\mathrm{VAT}$ & $1.0(0.88-1.14)$ & 0.99 & $1.03(0.93-1.13)$ & 0.61 & $1.02(0.94-1.10)$ & 0.64 & \\
\hline
\end{tabular}

BMI indicates body mass index; VAT, visceral adipose tissue

*Multivariable (MV) Model includes age, sex (in overall model only), cohort (Third Generation or Omni), accelerometer wear time (valid days of use), light PA minutes/day, MVPA minutes/day, current smoking status and drinks per week. 
Supplementary Table 2: Multivariable-adjusted linear regressions examining the associations between 30 minutes/day increment of physical activity and log-adjusted aminotransferase levels.*

\begin{tabular}{|c|c|c|c|c|}
\hline & \multicolumn{2}{|c|}{ Log-AST } & \multicolumn{2}{|l|}{ Log-ALT } \\
\hline & $\begin{array}{l}\text { Beta Coefficient } \\
(95 \% \mathrm{Cl})\end{array}$ & P Value & $\begin{array}{l}\text { Beta Coefficient } \\
(95 \% \mathrm{Cl})\end{array}$ & P Value \\
\hline \multicolumn{5}{|l|}{ MVPA (min/day) } \\
\hline $\begin{array}{l}\text { Multivariable (MV) } \\
\text { Model }^{\star \star}\end{array}$ & $0.17(0.08-0.26)$ & 0.0002 & $-0.04(-0.12-0.05)$ & 0.41 \\
\hline $\mathrm{MV}+\mathrm{BMI}$ & $0.17(0.08-0.26)$ & 0.0002 & $0.02(-0.06-0.10)$ & 0.62 \\
\hline $\mathrm{MV}+\mathrm{VAT}$ & $0.18(0.09-0.27)$ & 0.0001 & $+0.04(-0.04-0.13)$ & 0.30 \\
\hline \multicolumn{5}{|c|}{ MVPA Bouts (min/day) ${ }^{\star \star \star}$} \\
\hline $\begin{array}{l}\text { Multivariable (MV) } \\
\text { Model }^{\star \star}\end{array}$ & $0.29(0.15-0.42)$ & $<0.0001$ & $-0.09(-0.21-0.04)$ & 0.18 \\
\hline $\mathrm{MV}+\mathrm{BMI}$ & $0.30(0.16-0.43)$ & $<0.0001$ & $-0.01(-0.13-0.12)$ & 0.91 \\
\hline $\mathrm{MV}+\mathrm{VAT}$ & $0.30(0.16-0.43)$ & $<0.0001$ & $0.00(-0.12-0.13)$ & 0.95 \\
\hline \multicolumn{5}{|l|}{ Light (min/day) } \\
\hline $\begin{array}{l}\text { Multivariable (MV) } \\
\text { Model }^{\star \star}\end{array}$ & $0.03(-0.01-0.06)$ & 0.16 & $0.01(-0.03-0.04)$ & 0.71 \\
\hline $\mathrm{MV}+\mathrm{BMI}$ & $0.03(-0.01-0.06)$ & 0.16 & $0.01(-0.03-0.04)$ & 0.61 \\
\hline $\mathrm{MV}+\mathrm{VAT}$ & $0.03(-0.01-0.06)$ & 0.15 & $0.02(-0.02-0.05)$ & 0.33 \\
\hline \multicolumn{5}{|c|}{ 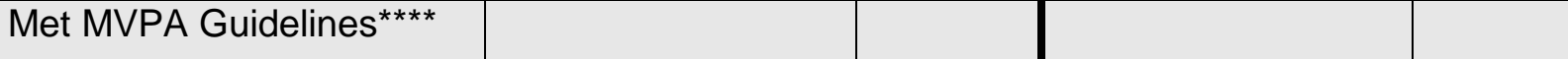 } \\
\hline $\begin{array}{l}\text { Multivariable (MV) } \\
\text { Model }^{\star \star}\end{array}$ & $0.17(0.04-0.29)$ & 0.008 & $-0.08(-0.20-0.03)$ & 0.17 \\
\hline $\mathrm{MV}+\mathrm{BMI}$ & $0.17(0.05-0.30)$ & 0.007 & $-0.00(-0.12-0.11)$ & 0.96 \\
\hline $\mathrm{MV}+\mathrm{VAT}$ & $0.17(0.05-0.30)$ & 0.007 & $0.02(-0.09-0.13)$ & 0.70 \\
\hline \multicolumn{5}{|c|}{ Met MVPA Guidelines ${ }^{\star \star \star \star \star}$} \\
\hline $\begin{array}{l}\text { Multivariable (MV) } \\
\text { Model** }^{\star *}\end{array}$ & $0.31(0.13-0.49)$ & 0.0007 & $-0.05(-0.22-0.12)$ & 0.54 \\
\hline $\mathrm{MV}+\mathrm{BMI}$ & $0.32(0.14-0.50)$ & 0.0006 & $0.03(-0.14-0.20)$ & 0.72 \\
\hline $\mathrm{MV}+\mathrm{VAT}$ & $0.32(0.14-0.50)$ & 0.0006 & $0.04(-0.13-0.20)$ & 0.66 \\
\hline
\end{tabular}


AST indicates aspartate aminotransferase; ALT, alanine aminotransferase; BMI, body mass index; VAT, visceral adipose tissue

*There were no significant sex interactions.

**Multivariable (MV) Model includes age, sex, cohort (Third Generation or Omni), accelerometer wear time (valid days of use), current smoking status and drinks per week.

${ }^{\star \star \star} A$ bout of MVPA is defined as at least 10 minutes of continuous MVPA allowing for a 2 minute interruption.

${ }^{* * * *}$ Met MVPA Guidelines is defined as participating in $\geq 150$ minutes of moderate-to-vigorous physical activity per week.

${ }^{* * * * *}$ Met MVPA Bout Guidelines is defined as participating in $\geq 150$ minutes of moderate-tovigorous physical activity per week accrued in bouts of at least 10 minutes.

Beta estimates provided above give standard deviation increase in log AST/ALT. 